This PDF is a selection from a published volume from the National Bureau of Economic Research

Volume Title: Health at Older Ages: The Causes and Consequences of Declining Disability among the Elderly

Volume Author/Editor: David M. Cutler and David A. Wise, editors

Volume Publisher: University of Chicago Press

Volume ISBN: 0-226-13231-5

Volume URL: http://www.nber.org/books/cutl08-1

Conference Date: October 8-11, 2004

Publication Date: January 2009

Chapter Title: Disability and Spending Growth

Chapter Author: Michael E. Chernew, Dana Goldman, Feng Pan, Baoping Shan

Chapter URL: http://www.nber.org/chapters/c11116

Chapter pages in book: (237 - 248) 


\title{
Disability and Spending Growth
}

\author{
Michael E. Chernew, Dana Goldman, \\ Feng Pan, and Baoping Shang
}

\begin{abstract}
Almost one of every six elderly Medicare beneficiaries suffers from some disability (U.S. Department of Health and Human Services 2004). Reductions in the prevalence and severity of disability could have dramatic effects on well-being; moreover, because the disabled spend more on health care than the nondisabled, lower rates of disability could reduce medical spending (Liu, Wall, and Wissoker 1997, Chan et al. 2002). For example, using data on Medicare beneficiaries between 1992 and 2000, Chernew et al. (2005) report that on average, the total medical care spending of people with one or two Activities of Daily Living (ADLs) was about twice that of nondisabled elderly. In contrast, people with five or more ADLs incurred four to five times the medical care spending of people without disability. Similarly, Cutler and Meara (1999) report that expenditure for persons with five or more activity limitations was nearly five times the amount incurred by those with Instrumental Activities of Daily Living (IADL) conditions.

Several studies in the health services literature posit that reduced disability levels in the future will lead to considerable cost savings. For example, Waidmann and Liu (2000) suggest that if disability rates continue
\end{abstract}

Michael E. Chernew is professor in the Department of Health Care Policy at Harvard Medical School, and a research associate of the National Bureau of Economic Research. Dana Goldman holds the RAND Chair in Health Economics and is Director of Health Economics at RAND. He is also a Professor of Health Services and Radiology at University of California, Los Angeles, and a research associate of the National Bureau of Economic Research. Feng Pan is a research associate at the United BioSource Corporation. Baoping Shang is a research associate in the Health Policy Center at the Urban Institute in Washington, D.C.

We are grateful for financial support from the National Institute on Aging grants P30 AG12810 and R01 AG19805, and the Mary Woodard Lasker Charitable Trust and Michael E. DeBakey Foundation. 
their current decline, the number of disabled elderly people will not grow either in absolute terms or relative to the size of the working-aged population, even in the face of the dramatic growth in the elderly population. Lubitz et al. (2003) show that the savings of improved health might offset the health care costs associated with longer life. Even though healthier people live longer, their total expected medical care expenses appear to be no greater than those for less healthy persons.

Yet Chernew et al. (2005) report that spending growth among the least disabled was faster than that among the more disabled. After adjustment for a range of covariates including demographic and health status, spending by the nondisabled and beneficiaries with only IADL disability grew 23 percent and 28 percent, respectively. This compares to a 10 percent increase for those with one or two ADLs, a 0.6 percent increase for those with three or four ADLs, and a 10 percent decrease for most disabled. As a result, the ratio of spending among the ADL disabled groups, relative to the nondisabled, declined over the study period. Thus, projections of cost savings based on the current pattern of spending by disability status may overstate the savings associated with improved disability.

This work expands upon the existing literature by exploring the spending trends by disability category for specific types of health care services. If a greater number of major procedures are performed on the less disabled, we would expect to see greater convergence in spending for inpatient services. If less disabled individuals are increasingly receiving expensive diagnostic tests or preventive services, relative to the more disabled, we would expect to see strong convergence in spending for physician and outpatient services. Greater use of medications among the less disabled, perhaps to manage chronic - though not necessarily disabling - conditions, would yield convergence in pharmaceutical spending across disability categories.

The pattern of results suggests that the convergence in spending is driven by reductions in spending for long-term care services. This reflects, in part, the effects of the Balanced Budget Act of 1997, which limited spending on long-term care. Because these services were disproportionately used by the most disabled, this led to a convergence of spending across disability groups. In addition to the effects of long-term care spending, we also find that pharmaceutical spending contributed to the convergence of spending across groups. In contrast to long-term care, where spending growth was constrained, spending on prescription medications was rapid in all disability groups. However, it was most rapid in the least disabled groups, leading to convergence in spending. Finally, we cannot reject the hypothesis that cost growth in inpatient care and provider/outpatient care was the same across disability categories. 


\subsection{Methods}

To estimate the trends in spending by disability group, we model total spending by Medicare beneficiaries (program spending plus beneficiary out-of-pocket spending) as a function of disability, other covariates (including disease burden), and a set of parameters. Estimates are conducted separately for total spending and four subcategories of spending: inpatient payments, outpatient and physician payments, prescription drug payments, and long-term care payments (hospice, home care, skilled nursing home, and facility).

Changes in Medicare spending over time will reflect either changes in the distribution of covariates (including disability) or changes in the coefficients that relate those covariates to spending. We estimate a nonlinear regression model of the form:

$$
\mathrm{E}\left[\text { spending }_{i t} \mid x_{i t}\right]=\exp \left(x_{i t}^{\prime} \beta\right)
$$

For services in which more than 90 percent of beneficiaries have some spending (total spending and provider/ outpatient spending), we estimate this model as a single-equation generalized linear model (GLM) where the variance of spending has a Gaussian distribution. In spending categories in which more than 10 percent of beneficiaries had no spending in a year, we estimate spending as a two part model, where the first part is a probit regression predicting positive spending (versus no spending in the category), and the second part is the GLM model described above, estimated on only those individuals with positive spending. Predicted spending by disability category and joint tests of the hypothesis that spending growth did not differ by disability category were based on analysis combining the estimates from both parts of the model.

To examine convergence of spending across disability categories, we include in all models interactions between covariates (most importantly disability category) and a linear time trend. We dropped the time interactions for those domains and disease states in which the estimates for total spending suggested the coefficients were stable over time. These dropped interaction terms include the interactions of time with: age, education, gender, race, region of residence, urban/rural, and marriage status. The dropping of the interaction terms signifies stability of the effects of these variables over time. The corresponding variables not interacted with time were retained in the model and were often important predictors of expenditures.

The GLM specification was based on specification tests of the total spending model. Relative to an Ordinary Least Squares (OLS) model and a similar nonlinear model that assumed a Gamma distribution instead of a Gaussian distribution, our model had lower mean average prediction errors using both a split sample approach and a set of models which were 
estimated on data from 1992 through a given year $(t)$ and then used results to predict expenditures in periods after $t$ through 2000. We estimated several such models using different years to define $t$.

Because disability is a marker of disease, a portion of the association between spending and disability may not be causal. Higher spending on individuals with disabilities may reflect efforts to treat the underlying disease that caused the disability. Moreover, if individuals with disabilities are disproportionately in poorer health (in unobserved ways) than other individuals, higher spending may reflect efforts to treat unrelated diseases.

\subsection{Data}

Our analysis is based on data from the 1992-2000 panels of the Medicare Current Beneficiary Survey (MCBS). The MCBS is a rotating panel survey in which a nationally representative sample of Medicare beneficiaries completes twelve interviews over three years. The survey is designed to ascertain utilization and expenditures for the Medicare population. Our analysis is confined to respondents enrolled in Medicare Part A and/or Part B and over sixty-five years of age. Beneficiaries eighty-five years of age or over are over-sampled. The MCBS provides demographic data including age, sex, race, and educational attainment. It also contains self-reported information on health, including the prevalence of various conditions, and measures of physical limitation in performing ADLs and IADLs.

\subsubsection{Measuring Costs}

Our measure of spending is based on Medicare claims data, linked to the MCBS and supplemented by respondent self-reports. Total spending includes both program spending and beneficiary out-of-pocket spending (Eppig and Chulis 1997). We include spending for all health care services (inpatient, outpatient, physician, pharmaceutical, nursing home, and home care). For services covered by Medicare, the data captures both the spending by Medicare, other payers including Medicaid, and by the beneficiary. Spending for services not covered by Medicare is based on self-reports and may be underreported. Spending by Medicare beneficiaries enrolled in HMOs is imputed based on measures of service utilization. All spending was converted to 2000 dollars using the consumer price index. The price index conversion will not influence relative spending across service types or disability groups.

\subsubsection{Measuring Disability}

Our measure of disability is derived from limitations in Activities of Daily Living (ADLs) and Instrumental Activities of Daily Living (IADLs). There are six ADLs: eating, bathing, dressing, transferring, walking, and toileting. There are eight IADLs: telephoning, using transportation, grocery shopping, personal shopping, housekeeping, chores, managing medica- 
tions, and managing money (Evashwick 2001). Activities of Daily Living and IADLs are measured on a five-point scale ranging from independent, requiring supervision, limited assistance, extensive assistance, or total dependence. Thus, the level of disability may be influenced not only by physical limitations, but also by the respondent's environment and social situation. For an ADL or IADL, we consider any respondent reporting that they require any supervision or assistance to suffer from that ADL. We then aggregate the disability measures into five categories commonly used in the literature: nondisabled, those with IADL only, with one or two ADLs, with three or four ADLs, and those with five or more ADLs (Liu, Wall, and Wissoker 1997; Cutler and Meara 1999; Manton and Gu 2001).

Beneficiaries residing in nursing homes do not report their disability status on a consistent basis. For this reason we treat nursing home residence as a separate category, which though related to disability, is not a direct measure of disability. Some community-dwelling elderly may suffer from greater disability than some nursing home residents.

\subsubsection{Measuring Disease}

Disease measured in the MCBS is based on self-report of whether the respondent has ever been told by a doctor that he had a list of important conditions. We capture the presence of diabetes, cancer, heart disease, stroke, Alzheimer's disease, hypertension, osteoarthritis, and lung disease using a series of indicator variables.

\subsubsection{Other Covariates}

We control for a range of demographic information such as age (dummy variables for ages sixty-five to sixty-nine, seventy to seventy-four, seventyfive to seventy-nine, eighty to eighty-four, eighty-five and over), gender, marriage status, race (white, black, Hispanic), education (less than eleven, twelve to fifteen, more than sixteen), region of residence (Midwest, West, Northeast, South, and other [Puerto Rico]). We also control for behavioral markers: current or former smoker; BMI category (obese, overweight, underweight); and supplemental insurance coverage (Part A only, Part B only, Medicaid, employer supplemental, private supplemental, and HMO).

\subsection{Results}

Each model yields two sets of coefficients. One set measures the effect of the relevant covariate in the base year (1992) and the other set measures how the effect of each covariate changes over time (the coefficients on the time interactions).

The base coefficients consistently indicate that spending rises as disability get worse (table 8.1). This is true for aggregate spending and for each category of spending. Spending on inpatient care exhibits the largest absolute differential in spending between the most disabled community-dwelling 


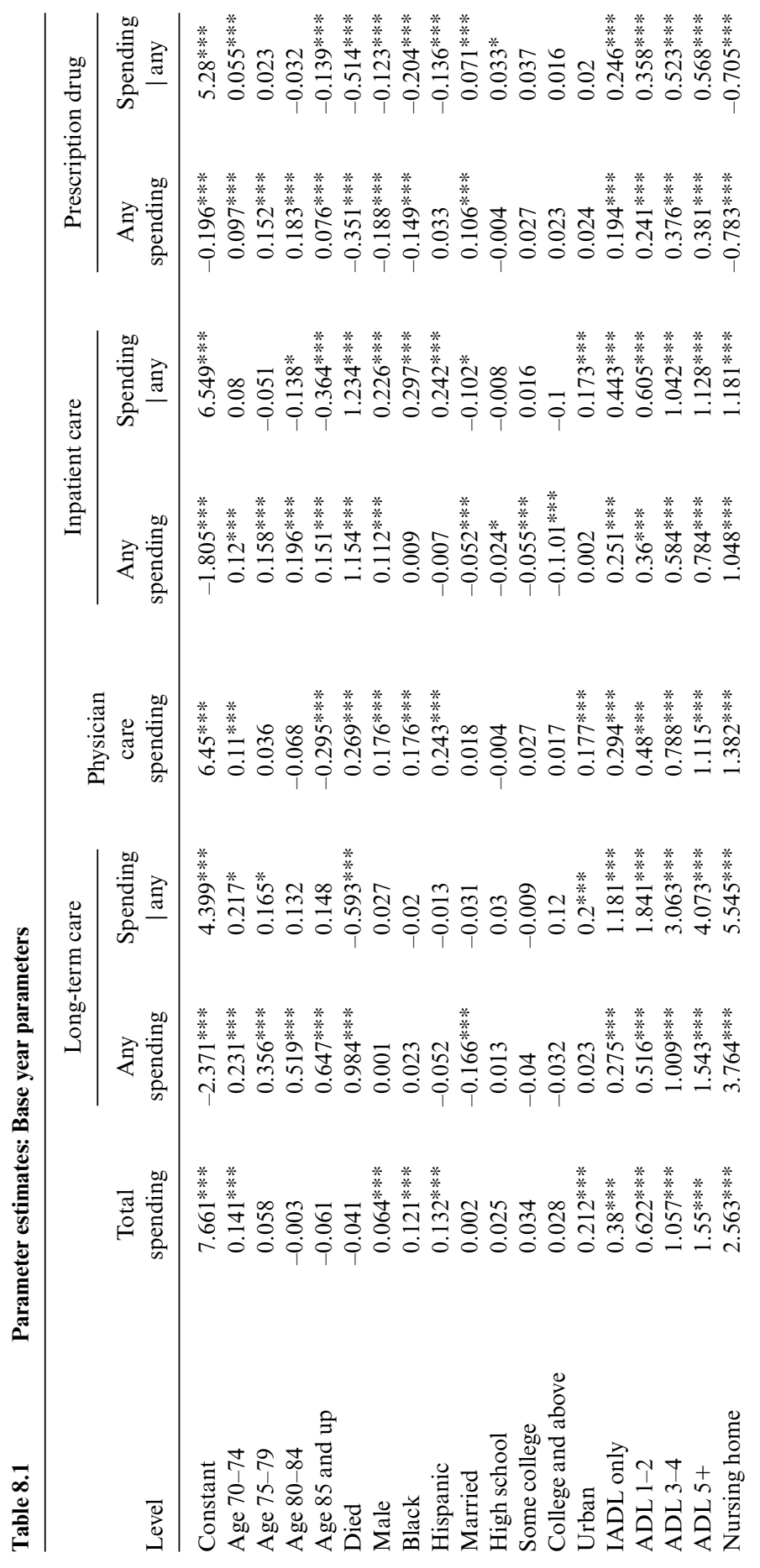




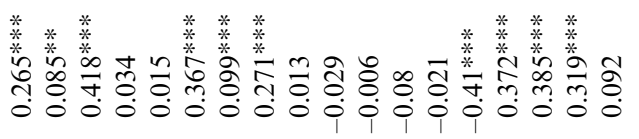

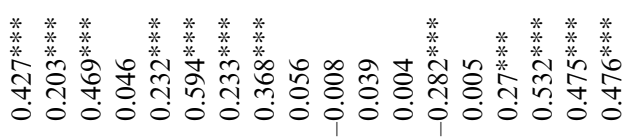

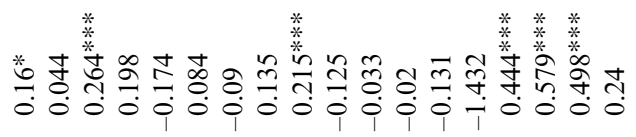

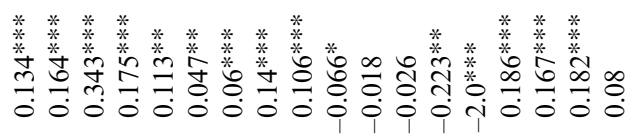

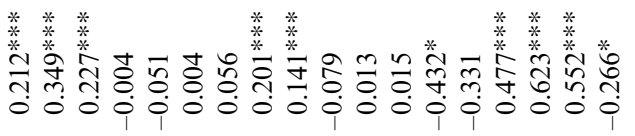

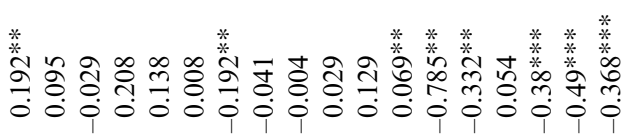

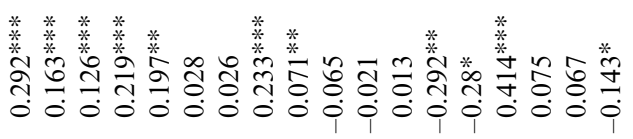

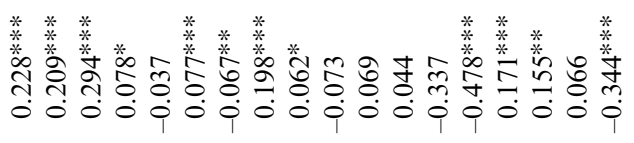

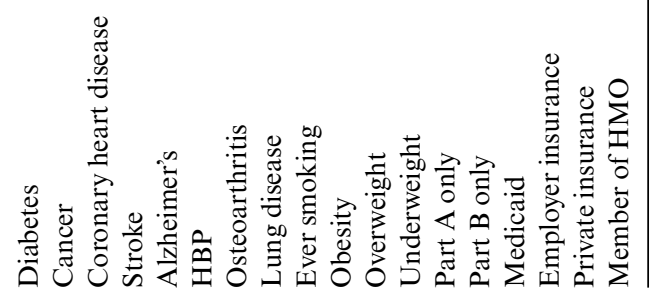

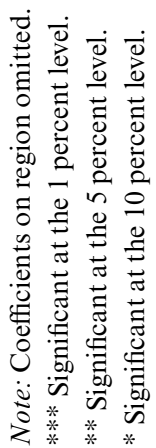


beneficiaries (more than five ADLs) and the nondisabled. This is followed closely by the differential for long-term care spending. Because the nondisabled spend so little on long-term care, the ratio of spending by beneficiaries with five or more ADLs, relative to the nondisabled, is greatest for long-term care spending. Specifically, in 1992 adjusted spending for the most disabled community dwelling elderly was over sixty-five times as much as adjusted spending for nondisabled community dwelling. Spending for prescription drugs is the least influenced by disability. The ratio of predicted spending on prescription medications for beneficiaries with five or more ADLs in the base year was only about twice that for the nondisabled.

Though the level of spending for the least disabled was much below that for the most disabled, the second set of coefficients from each model indicates that spending by the least disabled was growing at the fastest rate (table 8.2). As a result, the ratio of spending for the most disabled, relative to the least disabled was falling. Our estimates suggest that in 1992, spending by community-dwelling beneficiaries with five or more ADLs was 4.7 times greater than otherwise comparable nondisabled beneficiaries. That ratio fell to 3.3 by 2000 .

Examination of spending patterns reveals that most of this decline is driven by trends in long-term care spending. Estimated spending by the nondisabled remained low, but average annual spending by those with five or more ADLs, adjusted for demographic and other covariates, fell by about 7 percent. Closer examination of the data indicates that this was driven by a decline in home care spending following the Balanced Budget Act (BBA) of 1997. These findings reflect both a drop in the probability of any use and a drop in the amount of spending conditional on use. This is consistent with the study by Spector et al. (2004), which documents a reduction in home care spending overall following the BBA. The decline in home care spending was present for all disability groups, so the decline in the ratio of long-term care spending for the most disabled, relative to the least disabled was reasonably smooth, without a big break around the BBA. This is illustrated in figure 8.1, which reports the ratio of mean spending (unadjusted for covariates), for total long-term care spending and its two largest components, home care and care in short-term facilities. However, because long-term care is more salient for the disabled, the reduction in spending associated with long-term care drives the reduction in the relative spending by the most disabled group.

The estimates for physician services and inpatient services do not reveal a statistically significant differential in the spending trends between the most and least disabled. In fact, for inpatient care the estimates suggest that if other beneficiary traits remained unchanged from 1992, the relative spending gap between the most and least disabled community-dwelling elderly would have risen.

For pharmaceutical spending, growth was rapid among all categories of 


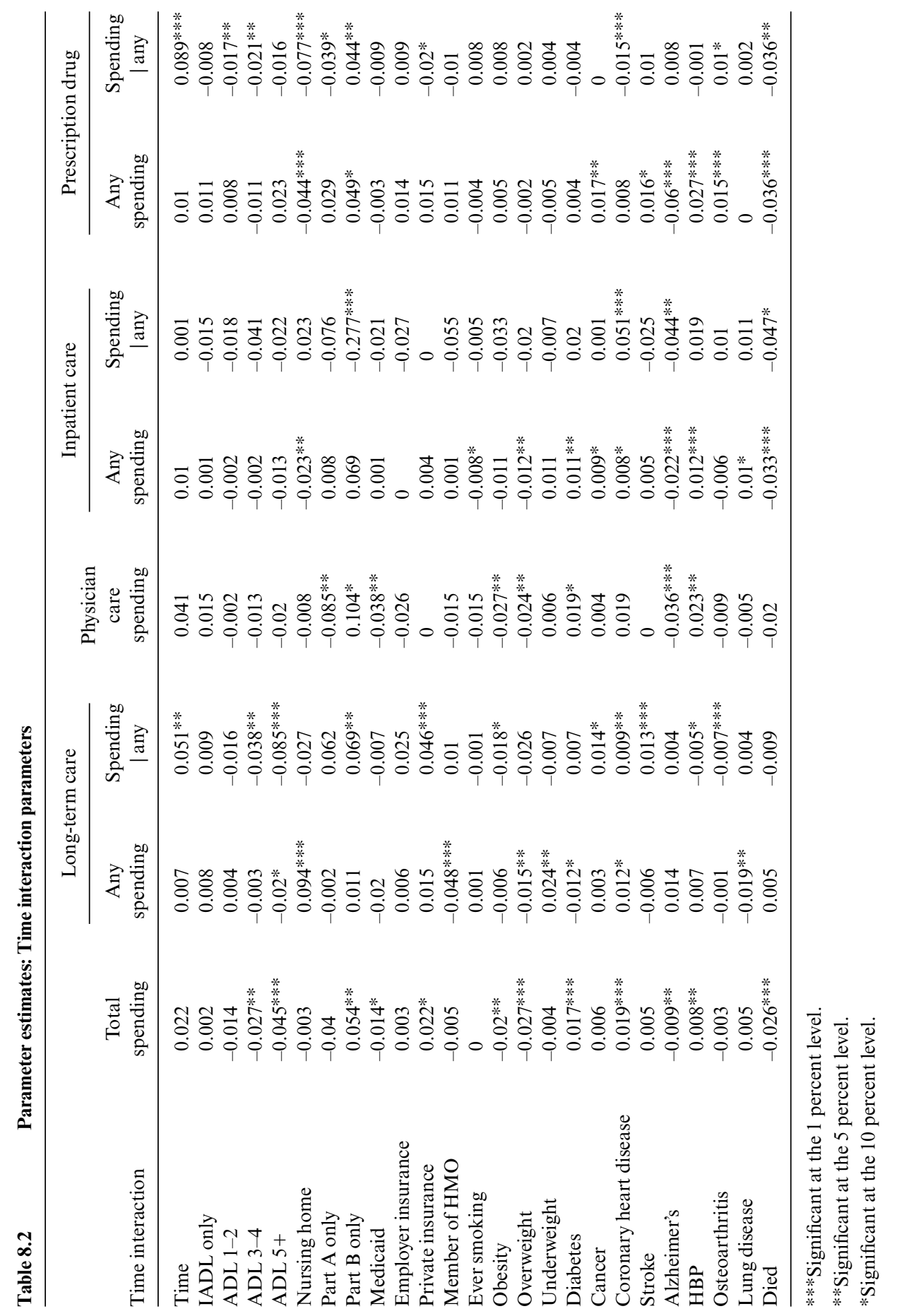




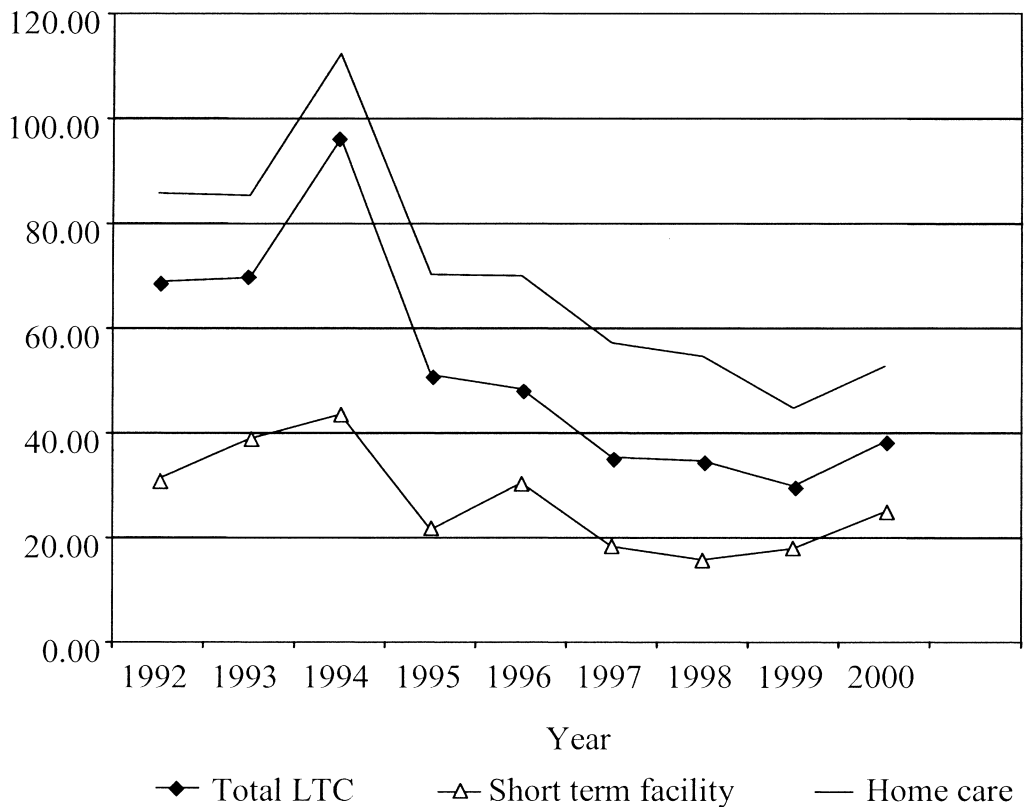

Fig. 8.1 Ratio of Long-Term Care spending, by type of Long-Term Care

disability, but most rapid among the least disabled. Although the coefficient on the interaction between five or more ADLs and time is not statistically significant, the point estimates suggests greater growth in spending for the nondisabled and the coefficients on the time interactions for the other two ADL disability groups are statistically significant. Although the relatively rapid growth in prescription drug spending among the nondisabled contributes to the decline in the ratio of spending by the most disabled, relative to the least disabled, the contribution of prescription drug spending to the overall decline is less than that of long-term care because the share of spending on pharmaceuticals is much lower on average ( 5 percent of spending for the most disabled and 14 percent for the nondisabled).

The results comparing the nondisabled to disability groups other than the most disabled reveal a similar pattern of spending ratios for the disabled relative to the nondisabled group. Overall, only the IADL-only group has very similar spending growth as the nondisabled group. In part this is because, like the nondisabled group, the IADL-only group has very limited spending on long-term care, which was the primary driver of the declining relative spending by the most disabled. 
The other ADL groups have slower spending growth in aggregate than the nondisabled (and IADL-only) group. Again, this reflects largely less spending on long-term care and somewhat slower growth in pharmaceutical spending relative to the less disabled groups.

\subsection{Conclusion}

During the 1990s, spending growth for the least disabled exceeded that by the most disabled. This resulted in a convergence of spending between the two groups of beneficiaries. If such a convergence continues, savings accruing to improved disability status may have less of an impact on overall spending than analysis of current spending patterns would suggest.

This analysis reveals that the convergence of spending reflects the reduction in long-term care spending following the BBA. While all disability groups experienced a decline in long-term care spending, the aggregate spending for the most disabled was most affected because long-term care spending is about a quarter of all spending for the most disabled group and less than 5 percent of spending for the nondisabled group.

A fundamental question is whether this convergence will continue. To a large extent the answer depends on decisions by policymakers. The Balanced Budget Act of 1997 was a major reason for the decline in long-term care spending, and thus an important contributor to the convergence in spending. If policymakers can hold the line on long-term care spending, one might expect to continue to observe slower relative spending growth among the most disabled. Moreover, it may be the case that technical innovations that drive spending growth are less salient for long-term care services, suggesting that pressures for increased spending on those services, per beneficiary, will be manageable.

In contrast, technical innovations related to physician, inpatient, and pharmaceutical services appear to affect beneficiaries in all disability groups. In fact, some innovations, such as those related to prescription drugs, were generally more salient for the least disabled group of beneficiaries. As these services consume a growing share of spending, we would forecast greater cost pressures. Like long-term care spending, spending for physician, inpatient, and pharmaceutical services will be sensitive to regulatory changes. Yet historically, constraining the growth of these services has appeared a more difficult task.

As we are faced with a growing number of Medicare beneficiaries, the challenge for policymakers will be to design payment systems and other regulations in a way as to promote efficient delivery of care and allocation of services. The political tradeoffs will be difficult to make and budgetary pressures great. Our analysis suggests that it is unlikely that improved disability status among elderly will eliminate the need for these tough choices. 


\section{References}

Chan, L., S. Beaver, R. F. Maclehose, A. Jha, M. Maciejewski, and J. N. Doctor. 2002. Disability and health care costs in the Medicare population. Archives of Physical Medicine \& Rehabilitation 83 (9): 1196-201.

Chernew, M., D. Goldman, F. Pan, and B. Shang. 2005. Disability and health care expenditures among Medicare beneficiaries. Health Affairs Web Exclusive, accessed at http://content.healthaffairs.org/webexclusives

Cutler, D. M., and E. Meara. 1999. The concentration of medical spending: An update. NBER Working Paper no. w7279.

Eppig, F., and G. S. Chulis. 1997. Matching MCBS and Medicare data: The best of both worlds. Health Care Financing Review 18 (3): 211-29.

Evashwick, C. J. 2001. The Continuum of Long-Term Care, Second Edition. Albany, NY: Delmar Thomson Learning.

Liu, K., S. Wall, and D. Wissoker. 1997. Disability and Medicare costs of elderly persons. Milbank Quarterly 75 (4): 461-93.

Lubitz, J., L. Cai, E. Kramarow, and H. Lentzner. 2003. Health, life expectancy, and health care spending among the elderly. New England Journal of Medicine 349 (11): 1048-55.

Manton, K. G., and X. Gu. 2001. Changes in the prevalence of chronic disability in the United States black and nonblack population above age 65 from 1982 to 1999. Proceedings of the National Academy of Sciences of the United States of America 98 (11): 6354-9.

Spector, W. D., J. W. Cohen, and I. Pesis-Katz. 2004. Home care before and after the Balanced Budget Act of 1997: Shifts in financing and services. Gerontologist 44 (1): 39-47.

U.S. Department of Health and Human Services. 2004. Health, United States 200: With chartbook on trends in the health of Americans (Health United States). Hyattsville, MD: CDC National Center for Health Statistics.

Waidmann, T. A., and K. Liu. 2000. Disability trends among elderly persons and implication for the future. Journals of Gerontology (B): Psychological Sciences and Social Sciences 55, no. 5, S298-307. 